\title{
Intermodal beat frequency stability in a polarization stabilized HeNe laser with an optical switch
}

\author{
Grzegorz Budzyń, ${ }^{* 1}$ Tomasz Podżorny, ${ }^{1}$ and Janusz Rzepka ${ }^{1}$ \\ ${ }^{1}$ Faculty of Electronics, Wroclaw University of Technology, Janiszewskiego 7, 50-370 Wroctaw,
}

Received August 01, 2013; accepted March 23, 2014; published March 31, 2014

\begin{abstract}
We investigated the behaviour of a frequency stabilized $1 \mathrm{~mW}$ two mode helium-neon laser incorporating an optical switch. In the paper we compare the stability of the intermodal frequency when the error signal is taken either from the main or parasite output of the tube. The possibility of using the constructed laser in a non-interferometric optical measurement device working with precision in the range of $1 \mathrm{ppm}$ is given.
\end{abstract}

Optical distance measurement methods can be, in general, divided into two categories: interferometric [1-2] and time of flight TOF. The methods from the first category are very accurate but difficult in use. The methods from the second category have limited resolution but are easy to use even over large distances. Recent improvements in signal processing allow using TOF methods with accuracies close to the industry standard range, i.e. below $10 \mu \mathrm{m}$ per meter. Such high accuracy, although requiring the use of high frequency electronic components, opens up a new important field of interest for TOF based instruments - calibration of different industry machines like lathes, milling machines, etc.

There are known two methods to measure the time of flight: direct measurement of time using a short light pulse and phase shift measurement of a modulated light beam. The former method is superior for measurement of longer distances. The latter seems more suitable in short to medium distance ranges [1]. The phase and frequency stability of the modulation signal is of indisputable importance in both methods [2].

There exist a couple of methods of modulation of the amplitude of laser light - mainly optical modulation and supply current modulation. One of the most promising ones is the use of natural intermodal beat frequency of a $\mathrm{HeNe}$ laser. In this method, high modulation frequencies are easily attainable (above $100 \mathrm{MHz}$, even above $1 \mathrm{GHz}$ ) with a modulation depth near to $100 \%$. In order to obtain beat frequency modulation it is enough to use a standard two-mode HeNe laser. Modulation frequencies exceeding $1 \mathrm{GHz}$ can be obtained with standard $<1 \mathrm{~mW}$ models available on the market (Lasos, MellesGriot). Because the natural intermodal beat frequency of the HeNe lasers is modest (>10ppm) [3-4], prior to the use in a distance meter application the laser frequency has to be stabilized [5].
In this paper we compare two construction methods of a two-mode HeNe laser with the use of an optical switch [6]. In the first method, the error signal is taken from the main mirror. In the second method, the error signal is taken from the parasite beam. We focus on the quantitative comparison of the stability of the intermodal beat frequency of constructed circuits.

For a two-mode laser, its longitudinal modes are placed within the gain curve of the laser (Fig. 1). The frequency difference between both modes is described as a Free Spectral Range FSR or intermodal beat frequency $v_{B}$ and, in the empty cavity, is defined as a ratio of the speed of light $\mathrm{c}$ and doubled cavity length $\mathrm{L}[7,5]$.

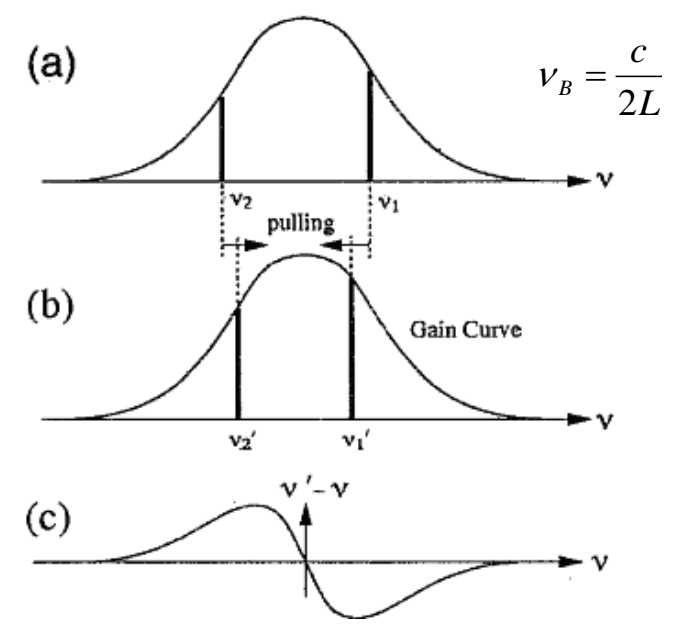

Fig. 1. Longitudinal modes within gain curve of the two-mode $\mathrm{HeNe}$ laser [3], $v_{\mathrm{B}}$-free spectral range, c-the speed of light, L-cavity length.

As shown by Araki et al. [2], the value of $v_{\mathrm{B}}$ in real lasers is influenced by the effect of frequency pulling. Because of this effect the $v_{B}$ value changes periodically with every $\lambda / 2$ cavity length expansion. The modified value of the $v_{B}$ ' can be modelled by equation [2]:

$$
v_{B}^{\prime}=v_{B}+\frac{c}{\pi \cdot \Delta v}\left[\gamma\left(v_{2}\right)\left(v_{0}-v_{2}\right)-\gamma\left(v_{1}\right)\left(v_{0}-v_{1}\right)\right]
$$

where $\gamma\left(v_{1}\right), \gamma\left(v_{2}\right)$ are laser gain curves for mode 1 and 2 respectively, $v_{0}$ is the centre frequency of the gain curve and $\Delta v$ is the half-width value of the gain curve. 
The measured changes of the beat frequency versus time were reported in some sources [1, 4, 8]. The variation of the beat frequency with a change in the cavity length has to be noticed.

Although the above relation does not describe the frequency pulling effect completely [2] but on its base an intermodal frequency stabilised laser can be constructed. Two stabilisation methods can be used: a frequency based and an amplitude based. The frequency based method, with direct control of beat frequency, seems at first more straightforward. In real application it causes many problems though: the detection circuit is complicated as high frequency modulated signals have to be detected and a stable source of the reference frequency has to be used.

The amplitude method is simpler. This method is based on the phenomena that during the laser tube warmup the position of the modes within the gain curve changes causing the above mentioned periodical changes of the beat frequency value but also periodical changes of the intensity difference between both modes [3]. The dependence between both effects allowed constructing a simple and effective method of both main and intermodal beat frequency stabilization [8]. In this method the modes intensity ratio is taken as the error signal for the stabilization loop. The desired situation (with the error signal equal to zero) is when mode intensities become equal.

The modification of this stabilization method based on the usage of an optical switch was shown in [6]. In this method the liquid crystal cell with an analysing polarizer is used as an optical switch (see Fig. 2), allowing to pass on the Detector only the polarization chosen by the Generator. The main advantage of using the optical switch is the simplified construction of the laser electronics and mechanics. The laser becomes less vulnerable to noises and long term changes caused by elements ageing and temperature. Both short and long term frequency stability of the laser are improved [9].

Two setups were constructed as it is shown in Fig. 2. In setup 2a) the error signal was taken from the main output of the laser while in setup 2b) the parasite beam was used. As a laser tube, a Lasos LGR7655 internal mirror tube was used. The optical power of the main output beam was around $1 \mathrm{~mW}$. The parasite beam was around 100 times weaker.

In the constructed lasers, a thermal heater was used as the main working element. The heaters were driven by error amplifiers which were in fact PID circuits. Trials were made both with an analogue Comparator/Error Amplifier path and a digital path with a signal processor. The obtained results were very similar although the usage of a signal processor gives more flexibility in parameters tuning.

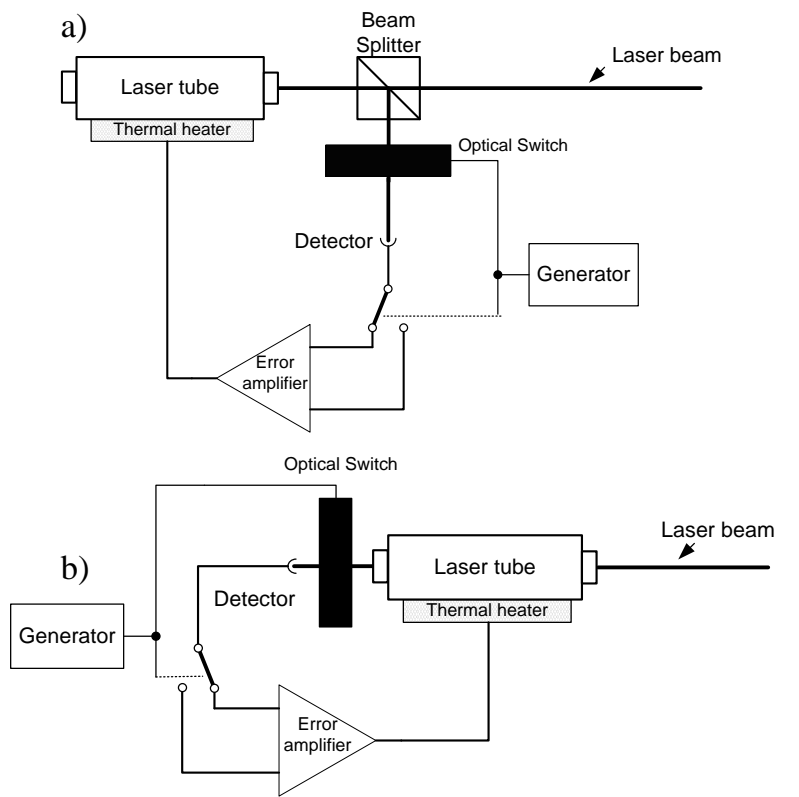

Fig. 2. Block diagram of a HeNe stabilized laser with error signal taken from the main beam (a) or the parasite beam (b).

The optical switch stabilized lasers described above were constructed and a series of measurements were performed. The beat frequency stability measurements were performed with a specialised "Allan Variance Meter" produced by Lasertex allowing automatic measurements of beat frequency at different averaging times.

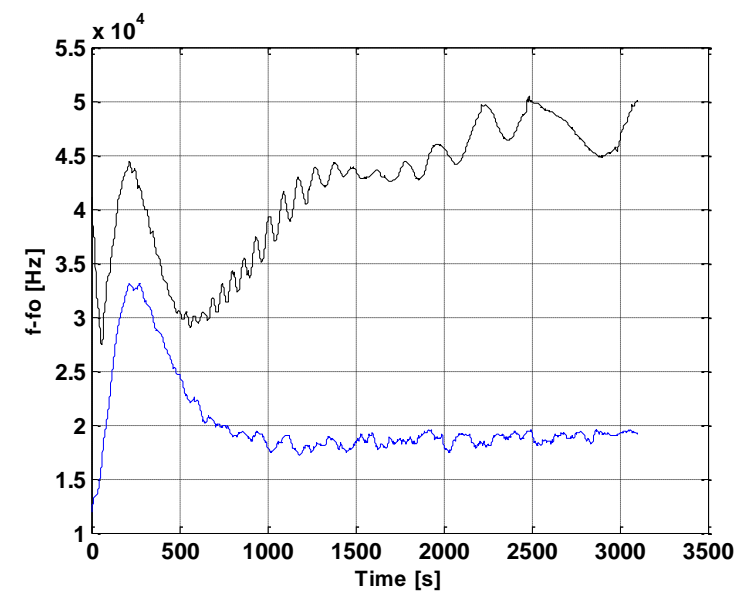

Fig. 3. Intermodal beat frequency during the first 3200 seconds after the stabilization loop lock. The upper line obtained in the circuit using the parasite beam. The lower line obtained in the circuit using the main output beam.

After the initial 15 minutes the beat frequency was becoming stable within $1-2 \mathrm{kHz}$ although for short observation times the stability was even better as it is shown in Fig. 4. By shorter observation time we mean still 
hundreds of a second, i.e. time sufficient for performing most measurements in practical conditions.

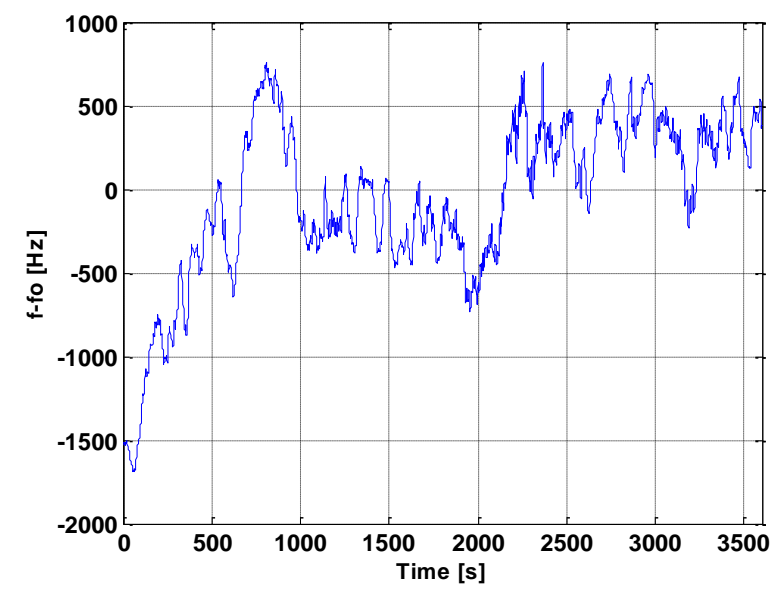

Fig. 4. Intermodal beat frequency measured 8 hours after the stabilization loop lock (setup 2a).

In order to characterise the behaviour of the laser more fully, we measured the beat frequency stability plot versus different averaging times (Fig. 5). At the averaging times longer than $10 \mathrm{~ms}$ the beat frequency stability was better than $10^{-6}$ or $1 \mathrm{ppm}$. The stability is even better at longer averaging times but we have never noticed it better than 0.06-0.07ppm.

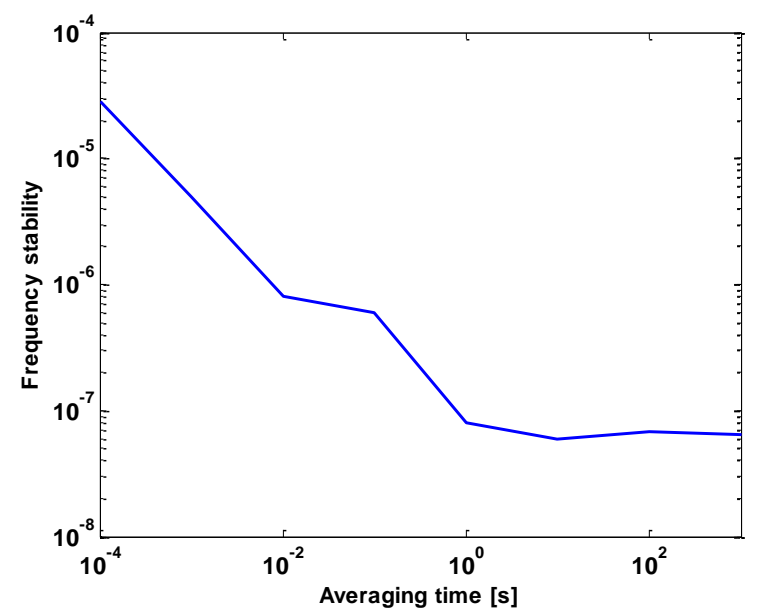

Fig. 5. Beat frequency stability of the setup using the main output beam versus averaging time ( $\operatorname{setup} 2 \mathrm{a})$.

From the performed measurements there can be drawn two important conclusions: 1) beat frequency stability is better when for the control loop a signal from the front mirror is taken; 2) in the tested setups it is not possible to obtain better stability of intermodal beat frequency than 0.06-0.07ppm even at very long averaging times.

We think that both effects can be explained by the facts shown by Anishenko [10] that the intermodal frequency has natural fluctuations and that this frequency depends on laser modes intensities [11]. The modes intensities, as shown by Yoshino [12], on the other hand, depend on the anisotropy of the multilayer mirrors used in the tested laser tube. Especially, the back mirror of the laser having a very high reflection coefficient seems to "modify" the passing beam in such a significant way that the control loop of the laser obtains false signals and overdrives the laser towards the wrong values of frequency stability.

In the paper we reported the stability of the intermodal beat frequency in a polarization stabilized $\mathrm{HeNe}$ laser with an optical switch. The intermodal beat frequency stability in the $1 \mathrm{~s}, 10 \mathrm{~s}$ and $100 \mathrm{~s}$ averaging points were better than $10^{-7}$, thus confirming our argument that the constructed stabilized $\mathrm{HeNe}$ can be used in noninterferometric optical distance measurement devices. The error caused by the laser itself would be lower than $1 \mathrm{ppm}$ making the device suitable in a broad range of different applications, even as a substitution to a classic laser interferometer. Because the accuracy of the classic laser interferometers not used in a vacuum depends almost solely on the accuracy of the air refraction coefficient compensation [13], the usage of the constructed laser in accurate measurements is possible. The only disadvantage is the lack of accuracy in dynamic measurements - at lower averaging periods the frequency stability is $10 \mathrm{ppm}$ or worse.

\section{References}

[1] K. Seta, T. O’Ishi, Appl. Opt. 29(3), 354 (1990)

[2] T. Araki, S. Yokoyama, N. Suzuki, Rev. Sci. Instr. 65(6), 1883 (1994).

[3] J. Rzepka, J. Pieńkowski, H. Pawołka, S. Sambor, Opt. Appl. XXVII(4), (1997).

[4] S. Yokoyama, T. Araki N. Suzuki, Appl. Opt. 30(3), 358 (1994).

[5] G. Wysocki, K.M. Abramski, Opt. Applic. XXXII(1-2), 207 (2002).

[6] J. Rzepka, P. Maltese, Proc. SPIE 3186, 162 (1997).

[7] L. Wang, et al., Chinese J. Lasers 34(9), 1198 (2010).

[8] M.-S. Kim, S.-W. Kim, Appl. Opt. 41(28), 5938 (2002).

[9] G. Budzyń, Optokomutacyjna stabilizacja lasera dwumodowego $(\mathrm{PhD}$ Thesis, Wrocław University of Technology, Wrocław 2006).

[10] M.L. Anishenko et al., Las. Phys. 1(5), 511 (1990).

[11] A. Lindberg, Am. J. Phys. 67(4), 350 (1991).

[12] T. Yoshino, Japn. J. Appl. Phys. 18(8), 1503 (1979).

[13] T. Podżorny, G. Budzyń, J. Rzepka, ICMEM 2012, 137 (2012).

[14] J.Y. Yeom, T.H. Yoon, Appl. Opt. 44, 266 (2005). 